\title{
Ecthyma gangrenosum on the face of a malnourished child with Pseudomonas sepsis: Simulating Cancrum oris
}

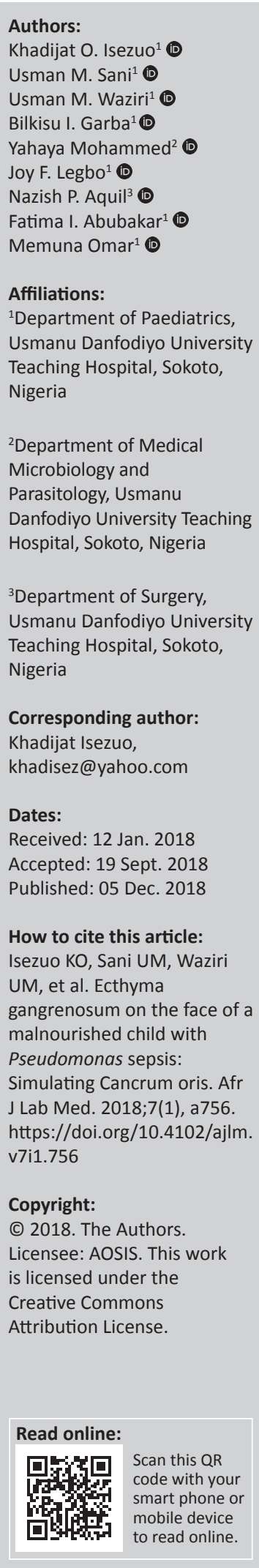

Introduction: Ecthyma gangrenosum (EG) is a cutaneous lesion commonly caused by Pseudomonas aeruginosa that involves mainly the lower limbs and gluteal region, seen more in immunosuppressed patients with neutropenia. Cancrum oris (Noma) is a gangrenous necrosis of the face that begins as a gingival ulcer and progresses rapidly to destroy contiguous tissues in malnourished children.

Case Presentation: This article reports a case of facial EG which was similar to Noma in a malnourished child: a 16-month old girl with fever, cough, weight loss, watery stool and swelling on right cheek. She was febrile, pale, wasted with bilateral pitting pedal oedema. She had a solitary circumscribed round necrotic lesion, with surrounding hyperaemia on the right malar area which extended to destroy the right ala nasi. No intra-oral rashes but she had left ear discharge. She received blood transfusion, antibiotics, antiseptic wound care and nutritional rehabilitation.

Management and Outcome: Swabs of the lesion and ear discharge both revealed Gramnegative bacilli and culture yielded P. aeruginosa. Retroviral, Mantoux and Gene Xpert tests were negative. She had moderate anaemia, normal white blood cell count, and neutropaenia. Parenteral ceftriazone was changed to ciprofloxacin based on sensitivity results and lack of clinical response. The wound healed with residual scarring and partial destruction of right ala nasi.

Discussion: Although this patient had facial necrosis to suggest Noma, she did not have initial oral involvement, and clinical features such as Pseudomonas sepsis and neutropaenia suggested EG. Facial necrosis in malnourished children may be due to EG.

\section{Introduction}

Ecthyma gangrenosum (EG) is a cutaneous lesion commonly caused by Pseudomonas aeruginosa, ${ }^{1,2}$ though it has been associated with other bacterial, viral and fungal aetiological agents. ${ }^{3,4,5}$ It occurs commonly in immunosuppression of various causes and is associated with neutropaenia. ${ }^{6}$ It may occur as a primary skin lesion which is the localised or non-septicaemic form or it may be the classical form which is a septicaemic illness associated with haematogenous spread of the causative bacterial organism. ${ }^{5,7}$

The typical appearance is that of a necrotic skin lesion with a central eschar surrounded by a hyperaemic halo. ${ }^{8}$ It commonly involves the lower limbs or genital area but may also involve other areas of the body occurring as solitary or multiple lesions. The sites commonly affected by EG lesions are the gluteal or perineal region (57\%), extremities (30\%), trunk (6\%), and face $(6 \%) .{ }^{9}$ Some have reported facial involvement in $12 \%$ of cases. ${ }^{4}$ Facial involvement has been reported to be common in neonates who are immunocompromised compared to older infants and children. ${ }^{10,11,12}$

Cancrum oris (Noma) is a severe oro-facial gangrene which occurs more in debilitated and malnourished patients. ${ }^{13}$ It usually starts as a gingival infected ulcer which rapidly becomes necrotic and spreads to produce extensive destruction of the tissues of the face in and around the oral cavity. Aetiology is usually polymicrobial by opportunistic organisms related to malnutrition and immune dysfunction. ${ }^{14}$ Some writers have described Noma-like necro-ulcerative lesions involving the face for which, if diagnosed early, intensive therapy may limit progression and confer better prognosis. ${ }^{15}$

In this report, we highlight a case of a malnourished child who presented with a necrotic ulcer involving the face which was similar to Noma but, on further review of the evolution of the ulcer 
and investigation of test results, was found more likely to be EG. We report the case to highlight that a necrotic facial ulcer presenting in a malnourished child may be EG and may mimic orofacial gangrene of Noma if not aggressively managed.

\section{Case presentation Ethical considerations}

Ethical approval for the study was obtained from the Research and Ethics Committee of Usmanu Danfodiyo University Teaching Hospital (Number: UDUTH/ HREC/2018/699). Written consent was obtained from the parents of the child. Confidentiality was maintained as well as privacy, as the identity of the child was known only to the authors who partook in her management. The clinical pictures taken were selectively occluded except for the aspect showing the lesion so as to prevent recognition of the patient.

\section{Method of data collection}

Data was collected by history-taking, clinical examination, clinical photographs and anthropometric measurements (weight, height and occipitofrontal circumference); venous blood samples were collected via aseptic technique for electrolytes, complete blood count, retroviral screening and blood culture. A nasogastric tube was used to collect samples of gastric washings. A sterile swab stick was used to collect samples of the lesion and ear discharge.

\section{Case report}

A 16-month old girl presented to Usmanu Danfodiyo University Teaching Hospital, Sokoto, in October 2016 with complaints of fever, cough and weight loss of 2 months, watery stool of 6 weeks, swelling on right cheek of a week's duration. The swelling initially started as a reddish rash which increased in size and darkened in colour. It was painless and not associated with discharge. She had poor nutritional and immunisation history. There was no preceding history of measles, though there was contact with other children who had pertussis.

On examination, she was febrile $\left(38.9^{\circ} \mathrm{C}\right)$, pale, wasted with bilateral pitting pedal oedema and fluffy hair. Her weight was 5 kilograms $(\mathrm{kg})$, length was $68 \mathrm{~cm}$ (Weight for length Z-score of $<-4$ standard deviation, expected value of -2 standard deviation to +2 standard deviation), occipitofrontal circumference was $40 \mathrm{~cm}$ (expected range of $47-48 \mathrm{~cm}$ ) and mid-arm circumference was $9.5 \mathrm{~cm}$ (expected normal value of $>14 \mathrm{~cm}$ ). She had a solitary circumscribed round necrotic lesion $(2 \mathrm{~cm} \times 2 \mathrm{~cm})$ with a central black eschar and surrounding hyperaemia on the right malar area which had minimal purulent discharge but was not foul-smelling (Figure 1). The central eschar sloughed off on the third day after admission to reveal a deep ulcer which had extended to destroy the right ala nasi (Figure 2). There was no intra-oral communication of the ulcer from the external surface. She did not have any intra-oral rashes, gingival ulcers or oral thrush.

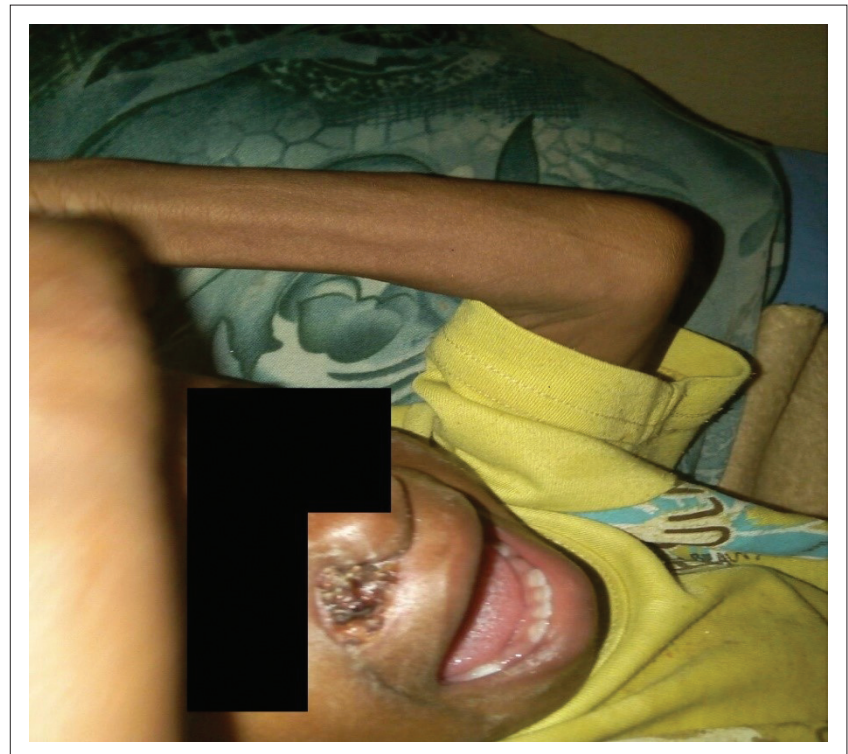

FIGURE 1: Necrotic eschar on second day after admission.

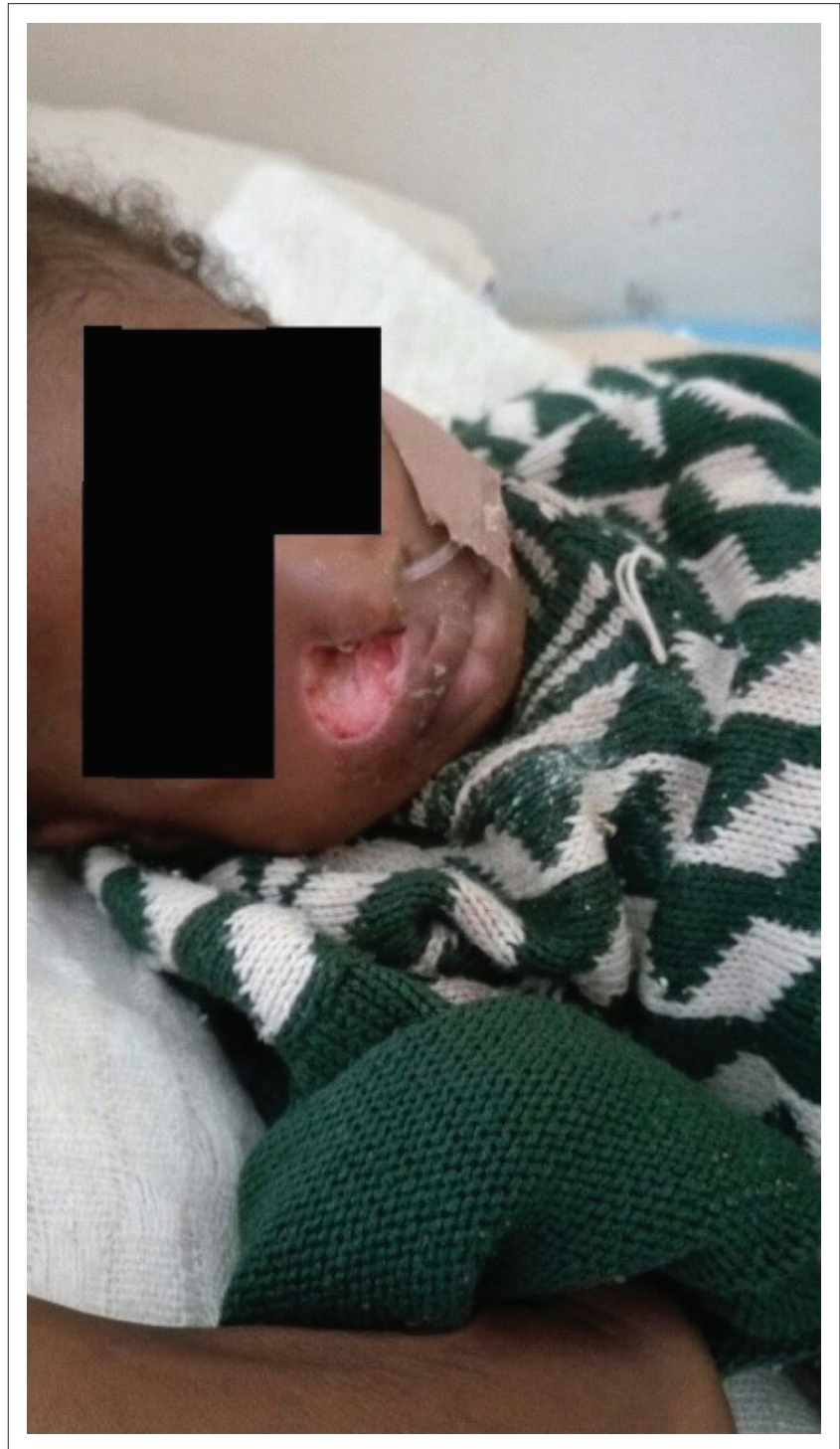

FIGURE 2: Deep ulcer extending to destroy the right ala nasi after sloughing of necrotic eschar. 
She developed purulent left ear discharge four days after admission. The external ear was normal; however, examination of the middle ear was not done as the child was not cooperative. Her pulse was 124 beats per minute and respiratory rate was 46 cycles per minute. She had vesicular breath sounds with reduced intensity and normal heart sounds. Abdominal examination revealed a non-tender hepatomegaly of $4 \mathrm{~cm}$. Neurological examination was normal.

\section{Management and outcome}

Swabs of the lesion and ear discharge showed pus cells and Gram-negative bacilli, while culture of both yielded P. aeruginosa. It was sensitive to ciprofloxacin ${ }^{++}$, ceftazidime $^{++}$, ofloxacin $^{++}$, and gentamicin ${ }^{++}$. Blood culture was however negative, although anaerobic culture was not done. Retroviral screening, Mantoux and Gene Xpert tests were all negative. Her packed cell volume was $22 \%$, which dropped to $18 \%$ on the seventh day, on account of which she was transfused packed red cells, resulting in post-transfusion volume of $32 \%$. Her total white cell count was $7 \times 10^{9} / \mathrm{L}$ (reference range $4-11 \times 10^{9} / \mathrm{L}$ ), with $16 \%$ constituting neutrophils (1120 cells, range: $2000-8000$ cells), and $84 \%$ lymphocytes (5880 cells, range: $1000-6000$ cells); platelets were $145 \times 10^{9} / \mathrm{L}$. The erythrocyte sediment rate was $28 \mathrm{~mm}$ fall/hr. The diagnosis was severe acute malnutrition (oedematous type) with complications of sepsis, severe anaemia and possibly evolving Cancrum oris. However, after further review of the patient's history, evolution of the lesion, in conjunction with the investigation results suggesting neutropaenia and isolation of $P$. aeruginosa from the lesion, EG involving the face was considered to be more likely.

She received blood transfusion, antibiotics, antiseptic wound care with normal saline soaks and dressing with honey. She also received nutritional rehabilitation in form of enriched pap and ready-to-use therapeutic food which is a nutritional rehabilitation diet manufactured mainly from peanuts. Electrolyte derangements (hypokalaemia and hyponatremia) were corrected with oral potassium supplements added to feeds and oral rehydration solution. Initial intravenous ceftriazone was changed to intravenous ciprofloxacin and intravenous gentamicin based on sensitivity results.

The ulcer healed remarkably but with residual scarring, including destruction of right ala nasi (Figure 3). Oedema subsided four days after admission and her weight rose gradually during the subsequent 2 weeks to $6.8 \mathrm{~kg}$. She spent about 3 weeks in the hospital. On a subsequent follow-up visit, she was doing well; however, the residual scar was still present, though much smaller. Caregivers defaulted on an appointment to see the co-managing plastic surgeon for possible repair of the residual scar.

\section{Discussion}

Ecthyma gangrenosum lesions were first described in 1897 in association with Pseudomonas septicaemia. ${ }^{16}$ EG usually begins as a haemorrhagic vesicle or pustule that gradually

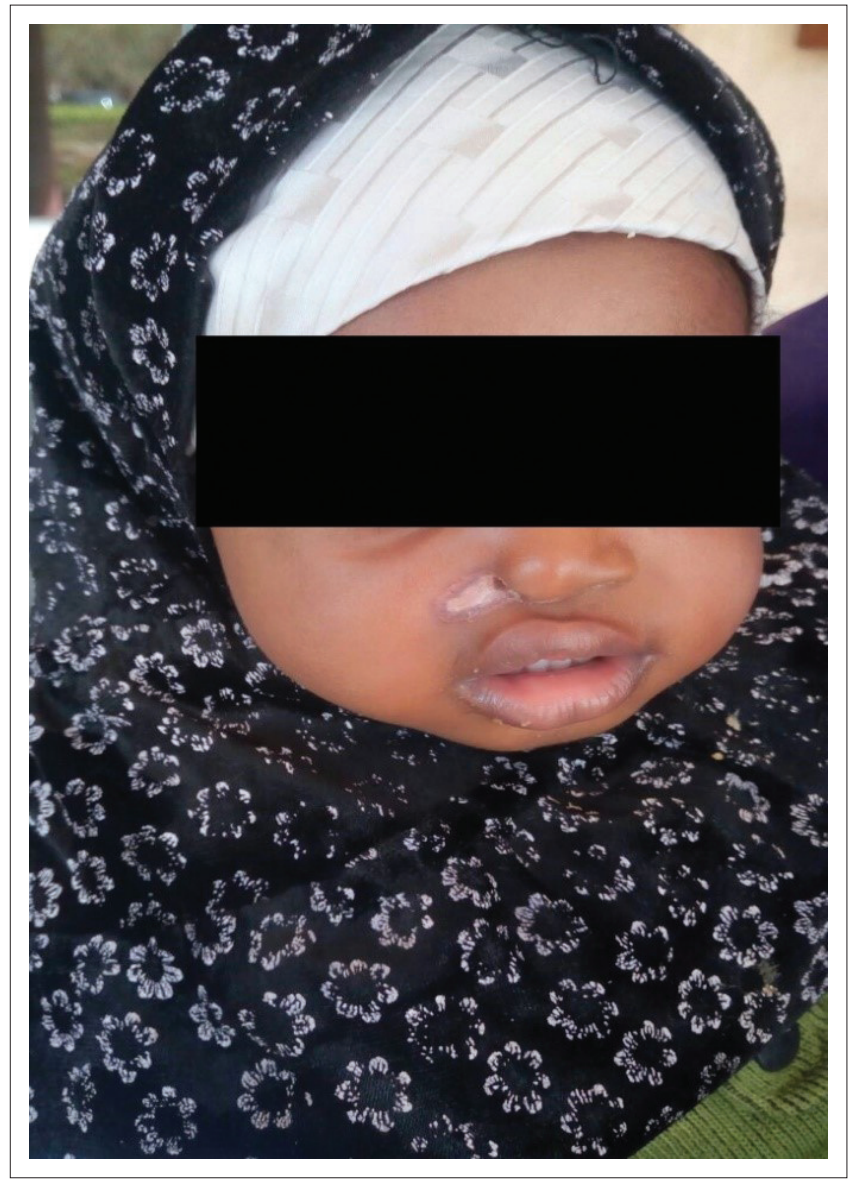

FIGURE 3: Residual scarring after healing of the ulcer.

evolves into a necrotic ulcer with eschar. This progression is because the bacteria invades the walls of the dermal and subdermal vessels leading to vascular damage and interruption of blood supply to the skin causing necrosis of the skin with eschar and ulcer formation. ${ }^{17}$ Although it has been recognised to be caused by a variety of other organisms, EG is most typically associated with severe systemic infection due to P. aeruginosa. ${ }^{17,18}$ It is most often seen in immunocompromised or neutropenic patients, though it can also occur in previously healthy individuals.

Our patient was immunocompromised as she was severely malnourished and she also had neutropaenia. ${ }^{19,20}$ The presence of acute otitis media caused by Pseudomonas in the child in addition to the skin manifestation of EG supported Pseudomonas sepsis even though the blood culture was negative. In a case report from Brazil of eight cases of EG in children, facial involvement was less common in the series. One of the cases was also a female infant with facial involvement and malnutrition similar to our case. ${ }^{21}$

Authors from India reported a case of a severely malnourished child with both EG involving the limbs caused by Pseudomonas and Noma; they suggested that both conditions can coexist with malnutrition as an underlying predisposing factor for both. ${ }^{22} \mathrm{~A}$ case was reported by Biddeci ${ }^{23}$ from Italy in a female infant who was previously healthy. She had a facial lesion similar to our patient which healed with a residual scar after 
treatment with anti-pseudomonal antibiotics based on a positive skin swab. Blood culture was also negative, similar to findings in our patient.

Noma neonatorum (a distinct entity from Noma in older children) which occurs in neonates, especially those with low birth weight and those born pre-term, is a rapidly progressive gangrenous lesion involving facial structures especially the mouth, nose and eyelids. It also involves the anal region and scrotum. ${ }^{24}$ It is also commonly associated with P. aeruginosa septicaemia. Although some authors have recently questioned distinguishing Noma in neonates from EG caused by Pseudomonas, ${ }^{11}$ Noma in older children is quite a distinct entity as it starts as a gingival ulcer and the ensuing gangrene rapidly spreads through muscles exposing the bone and teeth. ${ }^{13}$ Also, in Noma, the aetiological agents usually isolated include anaerobes like Prevotella intermedia, Fusobacterium nucleatum, Peptostreptococcus micros, Campylobacter and enteric Gram-negative organisms, while EG is mainly caused by Pseudomonas. ${ }^{13}$ However, these anaerobes cannot be completely ruled out in our case since anaerobic culture was not done.

The findings in the index case suggest that the child likely had EG rather than Noma given the evolution of the ulcerative process. However, without aggressive therapy or relatively early presentation of the patient, one may hypothesise that it could have spread, further simulating Noma. In conclusion, this patient had facial necrosis to suggest Noma; however, she did not have initial oral involvement, and clinical features suggested EG, which was supported by suspected Pseudomonas sepsis and neutropaenia. Facial necrosis in malnourished children may be due to EG.

\section{Acknowledgements Competing interests}

The authors declare that they have no financial or personal relationships that may have inappropriately influenced them in writing this article.

\section{Sources of support}

None.

\section{Authors' contributions}

K.O.I. conceptualised the report and wrote the initial draft. K.O.I., U.M.S., U.M.W. and B.I.G. were responsible for project design and reviewed the initial draft. Y.M. performed most of the experiments. J.F.L., N.P.A., F.I.A. and M.O. made conceptual contributions and performed some of the experiments.

\section{References}

1. Chan YH, Chong CY, Puthucheary J, Loh TF. Ecthyma gangrenosum: A manifestation of Pseudomonas sepsis in three paediatric patients. Singapore Med J. 2006;47:10801083.

2. Fang LC, Peng CC, Chi H, Lee KS, Chiu NC. Pseudomonas aeruginosa sepsis with ecthyma gangrenosum and pseudomembranous pharyngolaryngitis in a 5-monthold boy. J Microbiol Immunol Infect. 2014;47:158-161. https://doi.org/10.1016/j. jmii.2012.05.010

3. Reich $H$, Williams FD, Naik N, Honig P, Yan A. Nonpseudomonal ecthyma gangrenosum. J Am Acad Dermatol. 2004;50:S114-117. https://doi.org/10.1016/j. jaad.2003.09.019

4. Zomorrodi A, Wald E. Ecthyma gangrenosum: Considerations in a previously healthy child. Pediatr Infect Dis J. 2002;21:1161-1164. https://doi.org/10.1097 /00006454-200212000-00016

5. Pathak $A$, Singh $P$, Yadav $Y$, Dhaneria $M$. Ecthyma gangrenosum in a neonate: Not always pseudomonas. BMJ Case Rep [serial online]. 2013 [cited 2017 Dec 07]. Available from: http://casereports.bmj.com/content/2013/bcr-2013009287.full.pdf

6. Bozkurt I, Yuksel E, Sunbul M. Ecthyma gangrenosum in a previously healthy patient. Indian Dermatol Online J. 2015;6:336-338. https://doi.org/10.4103/2229 5178.164479

7. El Baze P, Thyss A, Vinti H, Deville A, Dellamonica P, Ortonne JP. A study of nineteen immunocompromised patients with extensive skin lesions caused by Pseudomonas aeruginosa with and without bacteremia. Acta Derm Venereol. Pseudomonas aerugi

8. Dorff GJ, Geimer NF, Rosenthal DR, Rytel MW. Pseudomonas septicemia. Illustrated evolution of its skin lesion. Arch Intern Med. 1971;128:591-595. https://doi.org/10.1001/archinte.1971.00310220099014

9. Elmariah $\mathrm{S}$, Ubriani R, James W, Kageyama N, Fish F. Ecthyma gangrenosum [homepage on the Internet]. [cited 2017 Dec 10]. Available from: http://www. emedicine.com/derm/topic539.htm

10. Foca MD. Pseudomonas aeruginosa infections in the neonatal intensive care unit Semin Perinatol. 2002;26:332-339. https://doi.org/10.1053/sper.2002.36266

11. Freeman AF, Mancini AJ, Yogev R. Is noma neonatorum a presentation of ecthyma gangrenosum in the newborn? Pediatr Infect Dis J. 2002;21:83-85. https://doi. org/10.1097/00006454-200201000-00025

12. Parikh TB, Nanavati RN, Udani RH. Noma neonatorum. Indian J Pediatr. 2006;73:439-440. https://doi.org/10.1007/BF02758572

13. Enwonwu CO, Falkler WA, Jr., Phillips RS. Noma (cancrum oris). Lancet. 2006;368:147-156. https://doi.org/10.1016/S0140-6736(06)69004-1

14. Raimondi F, Veropalumbo C, Coppola C, et al. Noma Neonatorum from multidrugresistant Pseudomonas aeruginosa: An underestimated threat? J Pediatric Infect Dis Soc. 2015;4:e25-e27. https://doi.org/10.1093/jpids/piu072

15. Nash ES, Cheng LHH, Smart K. Cancrum oris-like lesions. Br J Oral Maxillofac Surg. 1991;29:51-53. https://doi.org/10.1016/0266-4356(91)90176-6

16. Ecthyma gangrenosum [homepage on the Internet]. [cited 2017 Dec 10]. Available from: https://emedicine.medscape.com/article/1053997-overview.

17. Duffill M. Ecthyma gangrenosum [homepage on the Internet]. New Zealand: DermNet. [cited 2017 Dec 10]. Available from: https://www.dermnetnz.org/ topics/ecthyma-gangrenosum/

18. Bodey GP, Bolivar R, Fainstein V, Jadeja L. Infections caused by Pseudomonas aeruginosa. Rev Infect Dis. 1983;5:279-313. https://doi.org/10.1093/ clinids/5.2.279

19. Schaible UE, Kaufmann SHE. Malnutrition and infection: Complex mechanisms and global impacts. PLoS Med. 2007;4:e115. https://doi.org/10.1371/journal. pmed.0040115

20. Segel GB, Halterman JS. Neutropenia in pediatric practice. Pediatr Rev. 2008;29:12-24. https://doi.org/10.1542/pir.29-1-12

21. Martínez-Longoria CA, Rosales-Solis GM, Ocampo-Garza J, Guerrero-González GA Ocampo-Candiani J. Ecthyma gangrenosum: A report of eight cases. Anais Brasileiros de Dermatologia. 2017;92:698-700. https://doi.org/10.1590/ abd1806-4841.20175580

22. Vaidyanathan S, Tullu MS, Lahiri KR, Deshmukh CT. Pseudomonas sepsis with Noma: An association? Indian J Med Sci. 2005;59:357-360. https://doi. org/10.4103/0019-5359.16653

23. Biddeci G, Cutrone $M$, Mattei I, Valerio E, Favot F. Ecthyma gangrenosum of the cheek in a 6-month-old infant. Arch Dis Child. 2015;100:55-56. https://doi. org/10.1136/archdischild-2014-306852

24. Ghosal SP, Sen Gupta PC, Mukherjee AK, Choudhury M, Dutta N, Sarkar AK. Noma neonatorum: Its aetiopathogenesis. Lancet. 1978;2:289-291. https://doi. org/10.1016/S0140-6736(78)91691-4 